\title{
Invasive pneumococcal disease at University Medical Centre Ljubljana before and after introduction of routine pneumococcal vaccination
}

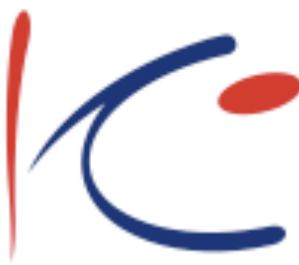

Marko Pokorn ${ }^{1}$, Katja Jarc ${ }^{2}$, Klavdija Strajnar ${ }^{2}$, Metka Paragi ${ }^{3}$

Department of Infectious Diseases, UMC Ljubljana, Slovenia

${ }^{2}$ Children's Hospital, UMC Ljubljana, Slovenia

${ }^{3}$ National Laboratory of Health, Environment and Food, Ljubljana, Slovenia

Background and aims

In Slovenia, surveillance of invasive pneumococcal disease (IPD) in children has been performed since 1993. All pneumococcal isolates are sent to the National Reference Laboratory for serotyping In 2015, the 10-valent pneumococcal conjugate vaccine (PCV10) was introduced $(2+1$ vaccination schedule with no catch-up) in the NIP on non-mandatory basis with $48.4 \%$ coverage in 2015 and $49.4 \%$ in 2016 . The aim of the study was to assess features of IPD and pneumococcal serotypes in children $<15$ years of age treated at the University Medical Centre Ljubljana (UMCL) in a two-year period before and after introduction of PCV10 vaccination.

\section{Methods}

Data on demographic, clinical and laboratory features of IPD in children $<15$ years treated at UMCL from 2013 to 2016 were collected. All isolates were serotyped and antimicrobial resistance was assessed.

\section{Figure 1. Number of IPD cases}

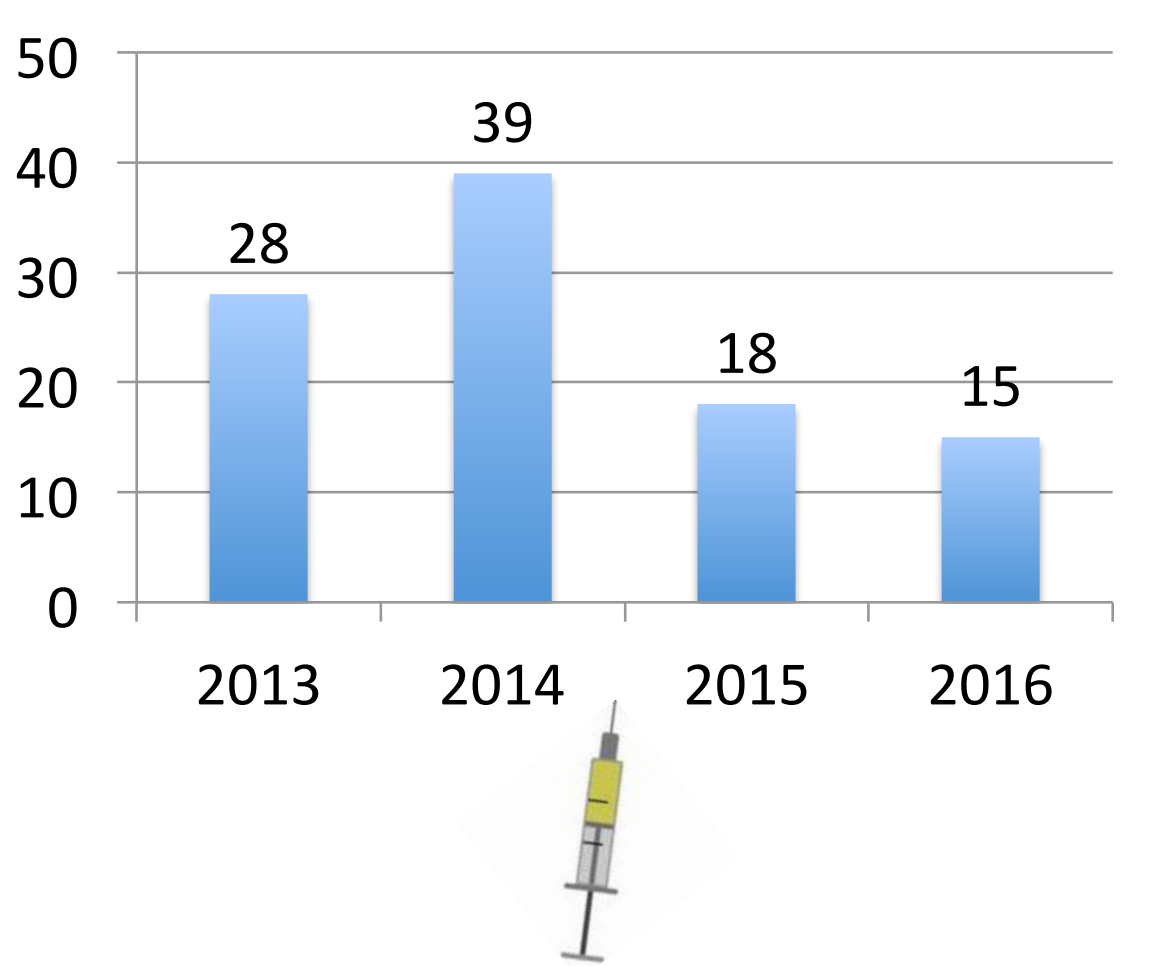

\section{Results}

Throughout the study period there were 100 IPD cases, 67 in 2013/14 and 33 in 2015/16 After PCV10 introduction, there was a $85 \%$ reduction of IPD cases in infants (13 vs. 2), followed by $75 \%$ in $24-59$ month olds ( $20 \mathrm{vs}$. $5)$ and a $24 \%$ in those $12-23$ months old (21 vs. 16). The most common syndrome in both periods was bacteremia, followed by pneumonia. Among pneumococcal isolates, in both periods vaccine serotypes prevailed. An increase in serotype 3,6A and 19A cases was not observed. The majority of isolates were penicillin-susceptible $(87 \%$ in $2013 / 14$, $90 \%$ in $2015 / 16)$, the remaining demonstrated intermediate resistance. All isolates were sensitive to cefotaxime. In 2013/14, 75\% of isolates were PCV10 and 91\% were PCV13 serotypes, whereas in $2015 / 16,76 \%$ of isolates were PCV10 and $88 \%$ were PCV13 serotypes.

Figure 2. IPD cases in various age groups, $2013 / 14$ vs. $2015 / 16$

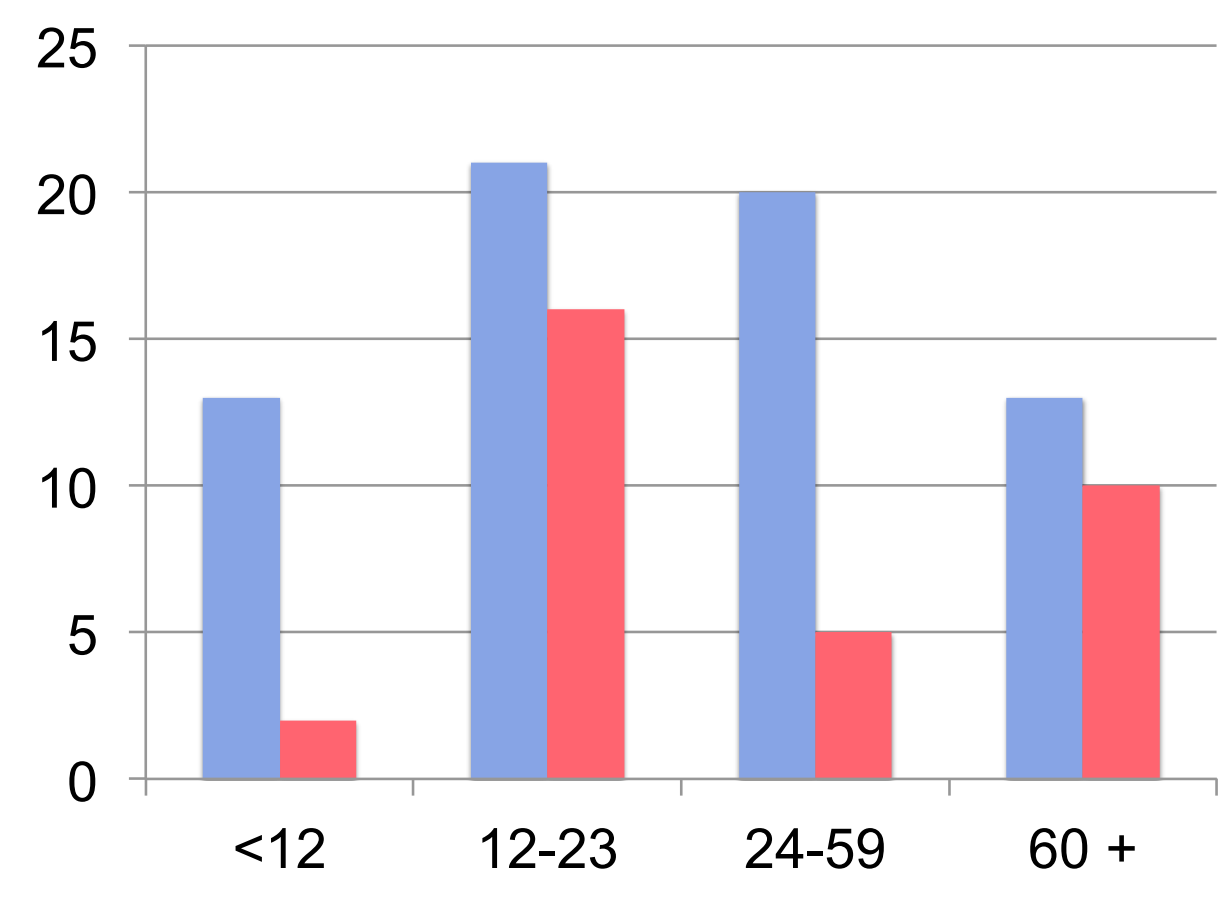

Table 1. Number of IPD syndromes

\begin{tabular}{|c|c|c|}
\hline Syndrome & $\mathbf{2 0 1 3 / 1 4}$ & $\mathbf{2 0 1 5 / 1 6}$ \\
\hline bacteremia & 25 & 17 \\
\hline pneumonia & 23 & 12 \\
\hline meningitis & 2 & 0 \\
\hline ethmoiditis & 9 & 2 \\
\hline AOM & 5 & 1 \\
\hline mastoiditis & 1 & 0 \\
\hline SA & 2 & 0 \\
\hline peritonitis & 0 & 1 \\
\hline
\end{tabular}

\section{Conclusions}

After PCV10 introduction in 2015 with roughly $50 \%$ vaccination coverage, a $50 \%$ decrease in number of IPD cases was observed at UMCL with vaccine serotypes predominating. The decrease in IPD numbers was most prominent in infants. The majority of isolates remain penicillin-susceptible. Further surveillance is needed to monitor changes in IPD epidemiology. Moreover, additional effort should be directed towards increasing pneumococcal vaccine uptake.

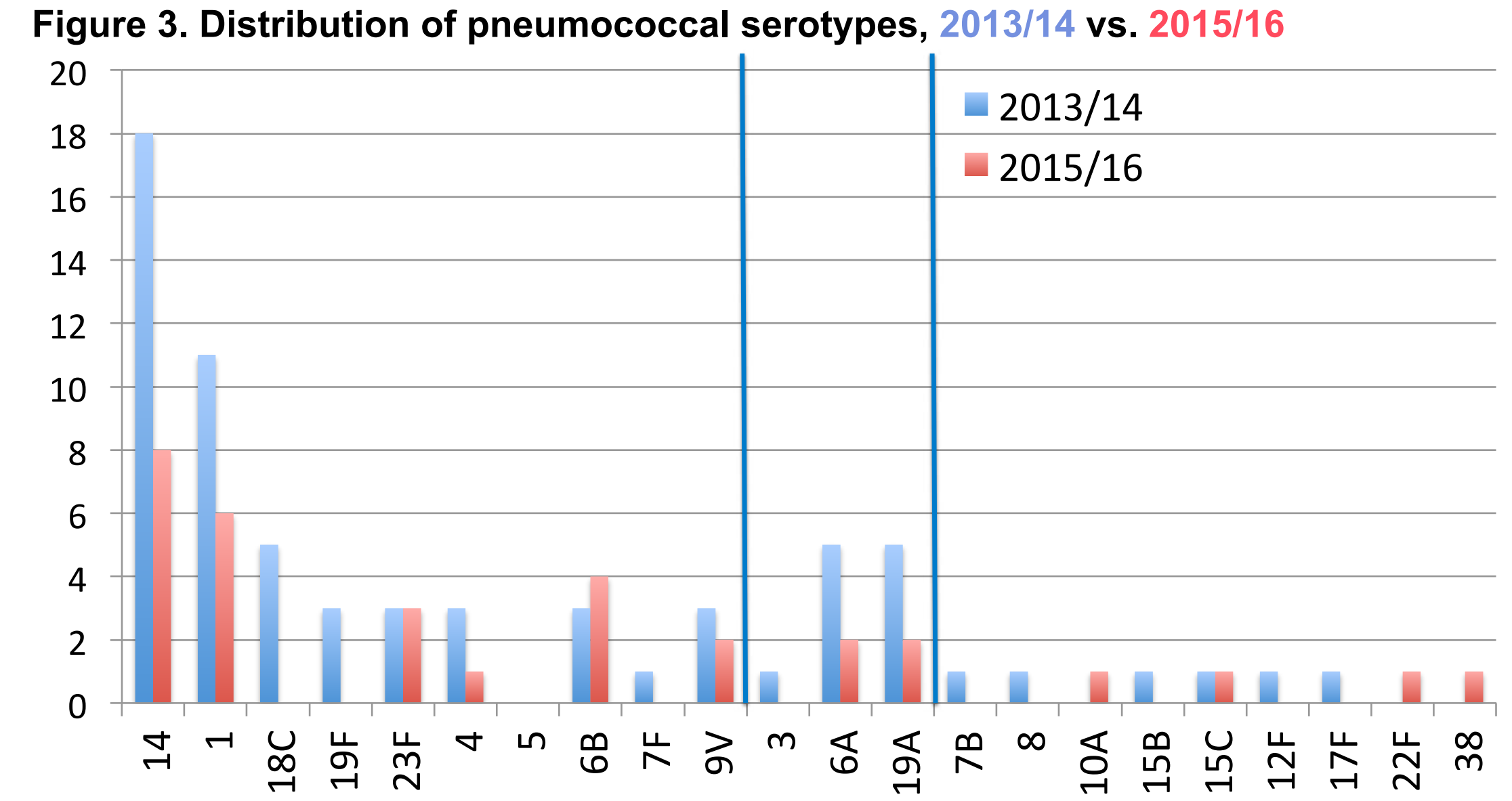

